\title{
RESHAPING FAECAL GUT MICROBIOTA COMPOSITION BY THE INTAKE OF TRANS-RESVERATROL AND QUERCETIN IN HIGH-FAT SUCROSE DIET-FED RATS
}

\author{
Etxeberria $\mathrm{U}^{1,2}$, Arias $\mathrm{N}^{3,5}$, Boqué $\mathrm{N}^{4}$, Macarulla $\mathrm{MT}^{3,5}$, Portillo $\mathrm{MP}^{3,5}$, Martínez $\mathrm{JA}^{1,2,5,{ }^{*}}$, Milagro $\mathrm{Fl}^{1,2,5}$
}

${ }^{1}$ Department of Nutrition, Food Science and Physiology, University of Navarra. C/Irunlarrea s/n, 31008 Pamplona, Spain

${ }^{2}$ Centre for Nutrition Research, University of Navarra. Irunlarrea St. E-31008 Pamplona, Spain

${ }^{3}$ Nutrition and Obesity group, Department of Nutrition and Food Sciences, Faculty of Pharmacy, University of the Basque Country (UPV/EHU), Paseo de la Universidad 7, 01006 Vitoria, Spain

${ }^{4}$ Nutrition and Health Research Group. Technological Center of Nutrition and Health (CTNS), TECNIO, CEIC S. Avinguda Universitat 1, 43204 Reus, Spain

${ }^{5}$ Physiopathology of Obesity and Nutrition, CIBERobn, Carlos III Health Research Institute, 28029 Madrid, Spain

*Corresponding author: Professor J. Alfredo Martínez

Department of Nutrition, Food Science and Physiology, University of Navarra. C/Irunlarrea s/n, 31 008 Pamplona, Spain. Tel.: +34 948425600 (80 6424)

E- mail address: jalfmtz@unav.es

\section{Abstract}

Diet-induced obesity is associated to an imbalance in the normal gut microbiota composition. Resveratrol and quercetin, widely known for their health beneficial properties, have low bioavailability and, when reach the colon, they are targets of the gut microbial ecosystem. Hence, the use of these molecules in obesity might be considered as a potential strategy to modulate intestinal bacterial composition. The purpose of this study was to determine whether trans-resveratrol and quercetin administration could counteract gut microbiota dysbiosis produced by high-fat sucrose diet (HFS) and in turn, improve gut health. Wistar rats were randomized into four groups fed a HFS diet supplemented or not with trans-resveratrol $(15 \mathrm{mg} / \mathrm{kg} \mathrm{BW} /$ day), quercetin (30 mg/kg BW/day) or a combination of both polyphenols at those doses. Administration of both polyphenols together prevented body-weight gain and reduced serum insulin levels. Moreover, individual supplementation of trans-resveratrol and quercetin effectively reduced serum insulin levels and insulin resistance. Quercetin supplementation generated a great impact on gut microbiota composition at different taxonomic levels, attenuating Firmicutes/Bacteroidetes ratio and inhibiting the growth of bacterial species previously associated to diet-induced obesity (Erysipelotrichaceae, Bacillus, Eubacterium 
cylindroides). Overall, the administration of quercetin was found to be effective in lessening HFS dietinduced gut microbiota dysbiosis. In contrast, trans-resveratrol supplementation alone or in combination with quercetin, scarcely modified the profile of gut bacteria, but acted at intestinal level altering the mRNA expression of tight-junction proteins (TJPs) and inflammation associated genes.

Keywords: flavonol; stilbene; gut bacteria; inflammation; obesity; intestinal permeability 


\section{Introduction}

Obesity is regarded as a major public health issue with an increasing worldwide prevalence [1]. Strong evidence support a direct link between intestinal dysbiosis, namely alterations of the gut microbial composition, and metabolic diseases encompassing obesity [2, 3] diabetes [4], liver disease $[5,6]$, cardiometabolic complications [7] and even cancer [8]. These findings have driven research interest to converge on making clear the intimate relationships among the gut microbiota, diet, host metabolism and the immune system [9]. In this context, the current use of genomic-based molecular techniques such as transcriptomics, metabolomics and metagenomics, together with the involvement of in vivo host models (i.e. germ-free animals) have allowed to boost the studies aiming to identify the most representative and significant gut bacterial groups or species in different disease conditions [10-12]. This specific characterization is helping to elucidate the role of gut microorganisms in the development of metabolic diseases [13]. Obesity has been characterized by a specific "bacterial trademark" represented by enlarged Firmicutes and reduced Bacteroidetes phyla [14], even if the association with the latter still remains being a matter of debate $[15,16]$. Furthermore, dysbiosis produced by high-caloric diets, i.e. high-fat diets $[17,18]$, has been also demonstrated to be susceptible to modulation by a variety of components such as probiotics and prebiotics [19]. Apparently, non-digestible polysaccharides could come to the aid of proliferating "friendly bacteria" and enhance microbial diversity in the gut [20], which might contribute to the amelioration of obesity and obesity-associated metabolic disorders [21].

Natural bioactive compounds are widely recognized to have potential biological properties [22, 23], such as anti-adiposity [24] and anti-diabetic effects $[25,26]$. Within the existing plant secondary metabolites, trans-resveratrol (stilbene) and quercetin (flavonoid) have been described to exert antiinflammatory, anti-obesity effects [27-29] and might be potential candidates for the amelioration of metabolic impairments (i.e. insulin resistance) associated to comorbidities of overweight or obesity $[30,31]$. Nevertheless, the health outcomes are intimately dependent on the dose and the form they are provided, and also on their bioavailability within the organism [32]. Overall, it is estimated that around $5-10 \%$ of the total dietary polyphenols are absorbed in the small intestine, while $90-95 \%$ of the total polyphenol intake reach the colonic region unabsorbed being the subject to enzymatic activities of gut microbial ecosystem producing diverse metabolites with a variety of physiological roles [33]. Although the metabolism of these bioactive compounds has been well reported [34-36], few studies have described its bioconversion by gut bacteria [37]. Nevertheless, in view of the direct contact and the dual interaction existing between these natural bioactive compounds and the colonic 
gut microbial ecosystem [38], possible composition modifications in intestinal bacteria might be expected as a result of the consumption of these compounds, which might ultimately contribute to functional modifications in the host.

Therefore, the present study was conducted to assess the potential of trans-resveratrol, quercetin, and a mixture of trans-resveratrol and quercetin supplementation to reverse HFS diet-induced gut microbiota dysbiosis in rats. Furthermore, this research work sought to analyse whether intestinal barrier integrity and inflammation might be affected as a consequence of polyphenol intake.

\section{Material and Methods}

\subsection{Animals, diets and serum biochemical parameters}

The experiment was performed with a sub-study of 23 Wistar rats obtained from Harlan Ibérica (Barcelona, Spain). Animals were single housed in polypropylene cages and kept in an isolated room with a constantly regulated temperature $\left(22 \pm 2{ }^{\circ} \mathrm{C}\right)$ under a $12: 12$-h artificial light/dark cycle Concisely, the artificial light/ dark cycle in this experiment was inverted, so that light was switched off at 9:00 am in the morning and switched on at 21:00 pm in the evening so that animals were expected to start eating as soon as the dark cycle began. Rats were fed a standard-chow diet (C; 2.9 $\mathrm{kcal} / \mathrm{g}$ ) from Harlan Iberica (ref. 2014, Barcelona, Spain) during an adaptation period that lasted six days. Afterwards, animals were randomly distributed into four groups and changed to a HFS commercial obesogenic diet (ref. D12451M) OpenSource, (HFS; $4.7 \mathrm{kcal} / \mathrm{g}$ ) for 6 weeks. The HFS diet contained $20 \%$ of energy as proteins, $35 \%$ of energy as carbohydrates (17 \% sucrose, $10 \%$ maltodextrin and $7 \%$ of corn starch) and $45 \%$ of energy as fat $(31.4 \%$ as saturated fats, $35.5 \%$ as monounsaturated fats, $33.1 \%$ as polyunsaturated fats). All animals had free access to food and water. The experimental groups were distributed as follows: the control group (HFS; $n=5)$, rats were fed the HFS diet; trans-resveratrol group (RSV; $n=6$ ), rats were supplemented with trans-resveratrol $15 \mathrm{mg} / \mathrm{Kg} \mathrm{BW} /$ day; quercetin group $(Q ; n=6)$, rats were supplemented with quercetin $30 \mathrm{mg} / \mathrm{kg} B W /$ day; and trans-resveratrol + quercetin group $(R S V+Q ; n=6)$, rats were treated with a mixture of trans-resveratrol $15 \mathrm{mg} / \mathrm{kg} \mathrm{BW/} \mathrm{day} \mathrm{and} \mathrm{quercetin} 30 \mathrm{mg} / \mathrm{kg} \mathrm{BW} /$ day. Polyphenols were daily incorporated to the powdered diet in quantities that ensured that each animal consumed the prescribed levels. Briefly, in a previous study, it was reported that resveratrol was degraded over the feeding period when it was mixed in the diet. Moreover, it was observed that rats started eating immediately upon daily diet replacement at the beginning of the dark period [39]. Thus, based on these assumptions and taking into account that resveratrol and quercetin have limited solubility in water, the compounds were added to the surface of the diet dissolved in ethanol. Variations in the amount of ethanol received by each animal were avoided as ethanol was added to the diet to reach 1 
$\mathrm{mL} / \mathrm{kg} \mathrm{BW/day}$. Although the volume of ethanol provided was negligible, control group was also given the same amount without natural compounds. Body weight and food intake were daily recorded. The doses of polyphenols were chosen based on previous studies [39-41]. Transresveratrol (> $98 \%$ purity) was supplied by Monteloeder (Elche, Spain) and quercetin ( $\geq 98 \%$ purity) by Sigma-Aldrich (St. Louis, MO, USA). All the experiments were performed in agreement with the Ethical Committee of the University of the Basque Country (document reference CUEID CEBA/30/2010), following the European regulations (European Convention- Strasburg 1986, Directive 2003/65/EC and Recommendation 2007/526/EC). Fasting serum glucose was measured using a Glucose oxidase/peroxidase kit (Ref. 110504, ByoSystems, Barcelona, Spain) and insulin levels were determined by using a specific ELISA Kit according to the protocol described by the manufacturer (Ref. 10-1250-01, Mercodia AB, Spain). Insulin resistance was assessed by the homeostasis model assessment of insulin resistance (HOMA-IR) formula [42]: [serum glucose levels (mmol/L) X insulin levels $(\mathrm{mU} / \mathrm{L})] / 22.5$. Inflammatory related markers were determined in serum with commercial ELISA Kits. TNF- $\alpha$ levels were determined with the TNF-alpha Platinum ELISA Kit (EBioScience, Viena Austria), lipopolysaccharide (LPS) levels by the use of Limulus Amebocyte Lysate (LAL) QCL-1000 ${ }^{\text {TM }}$ chromogenic assay (Lonza, Walkersville, MD) and LPS-binding protein (LBP) levels using the Mouse LBP ELISA Kit (Cell Sciences, Canton, MA).

\subsection{Faeces collection}

Fresh faecal samples were collected at the end of the intervention period early in the morning, prior to the overnight fasting. Each animal was taken one by one and located in a clean, single cage with the aim of obtaining faeces directly after defecation. A soft abdominal massage was also exerted to the animals in order to facilitate bowel movement and in turn, fresh faeces collection. Samples were gathered in $15 \mathrm{ml}$ Falcon tubes and immediately frozen at $-80^{\circ} \mathrm{C}$ for future analyses.

\subsection{Tissue collection}

At the end of the experimental period ( 6 weeks), animals were fasted overnight to assure on one hand, that all the animals were sacrificed in similar "feeding" conditions without the bias of the dietary treatment and on the other hand, to guarantee the suitability of biochemical values. Sacrifice was conducted under anaesthesia (chloral hydrate) by cardiac exsanguination between 9:00 and 12:00 am. Liver was collected, weighted and then stored frozen at $-80^{\circ} \mathrm{C}$. In all animals colonic mucosa was excised carefully by scratching and frozen immediately in liquid nitrogen for future RNA isolation. 


\subsection{DNA extraction, barcoded PCR and bacterial 16S rDNA pyrosequencing}

Faecal DNA was extracted using QIAamp DNA Stool Mini Kit (Qiagen, Hilden, Germany), following suppliers instructions. DNA was measured by NanoDrop ND-1000 spectrophotometer (Thermo Scientific, Delaware, USA). Samples for 454 pyrosequencing were amplified for the 16S rDNA hypervariable regions (V4 to V6) using 16S-0515F (5'-TGYCAGCMGCCGCGGTA-3') and 16S-1061R (5'TCACGRCACGAGCTGACG-3') universal primers. With the Roche 454 FLX chemistry, read lengths up to 550nt are achieved on PCR products which are in the range of 600-800nt, when using the offinstrument in amplicon mode. This mode favorizes the sequence quality of sequencing wells producing high signals. Since the $16 \mathrm{~S}$ molecules are very homogeneous and balanced in nucleotide composition, this mode produces better results than the shotgun mode, which is more tolerant to low signal beads, typically carrying GC- or extreme AT rich contents. The V4-V6 amplicon being 560nt in length for most bacteria, the full amplicon sequence can be recovered using a library construction protocol involving blunt ligation of sequencing adapters, the so-called Y-adapters, carrying DNA barcodes referred to as "multiplex identifiers" (MIDs) by the manufacturer. Sequencing on the 454 instrument is single-ended by nature, yet through the blunt ligation approach, approximately as many molecules are sequenced from the PCR $5^{\prime}$ end as there are molecules sequenced from the PCR 3' end.

Given the unit read length, the overlap between forward and reverse sequences is in the order of $90 \%$ and spanning the 3 variable regions. Thus, a sequence clustering approach using a de novo EST assembly process becomes possible without running the risk of generating chimeric sequence consensus. The Mira assembler [43] in EST mode can handle the kind of copy number variation corresponding with relative abundance differences observed among bacterial species in a metagenome sample. At the same time, this assembler can be made tolerant to single base changes often occurring between individual copies of the $16 \mathrm{~S}$ gene on a bacterial chromosome, which can be in the order of zero to two nucleotide changes over 100 nucleotides. The Mira assembler has a 454 specific error correction model and thus is capable of making the distinction between instrument basecall errors and intrinsic base-pair changes. Furthermore, combining forward and reverse sequences into a single consensus sequence achieves not only full length coverage but also increased sequence reliability, since the reverse sequences have high quality where the forward sequences exhibit quality drop-off and vice versa. Samples with high species diversity will generate a lower proportion of reads clustering than samples with more limited diversity. 


\subsubsection{Sequence post-processing}

The off-instrument 454 downstream processing software was used in amplicon mode to optimize read length as outlined above and to de-multiplex the libraries and trim off $3^{\prime}$ end adapter sequences, if any. The sequencing of the 23 amplicon libraries produced 16S rDNA reads ranging from 9780 to 71690 , generating read lengths from 479 to 520 nt, with a modal of 510nt.

The Mira assembler was used at 98\% similarity threshold, meaning sequences carrying up to 2 high quality differences out of 100 in any interval were allowed to assemble alongside with similar sequences. Singletons were carried forward into identification, provided their sequence length equalled at least 400nt. The same criterion was applied to contig consensus sequences produced. The proportion of reads producing clusters ranged from $36.1 \%$ to $58.5 \%$ on a per sample basis.

The singleton and consensus sequence were blasted using NCBI blastall version 2.2.22 in blastN mode, using an expectation value cut-off of $10^{\wedge}-5$ and requesting the 25 best hits produced against the RDP database release 10 update 29, which was curated for redundant entries representing identical sequence and entries with taxonomic classification of less than 4 levels down, counting from the "bacteria" taxon. These hits were duplicated according to the per contig sequence count and kept as-is for singletons, before being provided to the MEGAN classifier, version 4 [44]. MEGAN incorporates alpha rarefaction and assigns operational taxonomic units (OTUs) by comparing taxonomic information associated with equivalent best hits for each input sequence. Construction of libraries and sequencing were all provided as a custom service of Beckman Coulter Genomics.

\subsection{Gene expression in colonic mucosa}

Colonic mucosa RNA was extracted using All-Prep DNA/RNA Mini kit as described in the manufacturer's instructions (Qiagen, Germantown, Md., USA). RNA concentration and quality were measured with a Nanodrop ND-1000 spectrophotometer (Thermo Scientific). Subsequently RNA (2 $\mu \mathrm{g})$ was reverse-transcribed to cDNA using MMLV Moloney murine leukemia virus reverse transcriptase (Invitrogen) as described by the suppliers. RT-PCR assays were carried out following the manufacturer's instructions using an ABI PRISM 7000 HT Sequence Detection System, Taqman Universal Master Mix and predesigned TaqMan Assays-on-Demand (Applied Biosystems, Texas, USA). Interleukin 18 (IL-18), Rn01422083_m1; Nuclear factor kappa $\beta$ (NFk $\beta$ ), Rn00595794_m1; Tumor necrosis factor $\alpha$ (TNF- $\alpha$ ), Rn01525859_g1; Toll-like receptor 2 (TLR2), Rn02133647_s1; Tolllike receptor 4 (TLR4), Rn00569848_m1; Lipopolysaccharide binding protein (LBP), Rn00567985_m1; 
Myeloid differentiation primary response 88 (MyD88), Rn01640049_m1; Killer cell lectin-like receptor subfamily K, member 1 (NKG2D or Klrk1), Rn00591670_m1; Tight-junction protein 1 (TJP-1), Rn 02116071_s1; Tight-junction protein 2 (TJP-2), Rn01501483_m1; Occludin (Ocln), Rn 00580064_m1. mRNA levels were normalized with glyceraldehyde-3-phosphate dehydrogenase (GAPDH), Rn 99999916_s1 as a housekeeping gene (Applied Biosystems). All samples were analysed in triplicate. The relative expression level of each gene was calculated by the $2^{-\Delta \Delta \mathrm{Ct}}$ method.

\subsection{Short chain fatty acid (SCFA) analysis}

Short chain fatty acids were analysed in faeces. Briefly, $500 \mu \mathrm{L}$ of acid water, containing internal standard (propionic acid-d6, 98 atom \%D), was added to $25 \mathrm{mg}$ of each lyophilized sample. Overnight lyophilization was carried out protecting samples from light. Mixture was shaken for 1 minute. Afterwards, extraction was carried out with $500 \mu \mathrm{L}$ of diethyl ether followed by 1 minute of shaking and centrifugation at $4 \stackrel{\circ}{ } \mathrm{C}$ at $14000 \mathrm{rpm}$ for $10 \mathrm{~min}$. Supernatants were analysed by gas chromatography-mass spectrometry (GC/MS) in a 7890A Series gas chromatograph coupled to a 7000 GC/MS Triple Quad (Agilent Technologies, Santa Clara, U.S.A.). The chromatographic column was a J\&W Scientific HP-FFAP (30 m x $0.25 \mathrm{~mm}$ i.d., $0.25 \mu \mathrm{m}$ film) (Agilent Technologies). A volume of $1 \mu l$ of sample was automatically injected into a split/splitless inlet (in splitless mode), which was kept at $175^{\circ} \mathrm{C}$. Helium ( $99.999 \%$ purity) was used as a carrier gas, at a flow rate of $1 \mathrm{ml} / \mathrm{min}$ in constant flow mode. The oven program was set at an initial temperature of $90^{\circ} \mathrm{C}$, increased to $150^{\circ} \mathrm{C}$ at a rate of $12^{\circ} \mathrm{C} / \mathrm{min}$, then increased to $240^{\circ} \mathrm{C}$ at a rate of $20^{\circ} \mathrm{C} / \mathrm{min}$, and held at $240^{\circ} \mathrm{C}$ for $5 \mathrm{~min}$. Ionization was done by positive chemical ionization (PCI) with methane gas. SIM mode and GC-QqQ MassHunter software (Agilent Technologies) were used for data acquisition.

\subsection{Statistical analysis}

Results are expressed as the mean \pm standard error of the mean (SEM). Normality was tested by Shapiro- Wilk test. For parametric variables, One-Way ANOVA test followed by Dunnett post- hoc test was carried out. For non-parametric variables, statistical significance of differences among the groups was tested by non-parametric Kruskal- Wallis test followed by Mann Whitney U- test when $p<0.05$, Bonferroni correction test was applied as a correction for multiple comparisons. SPSS 15.0 software (Chicago, USA) was used to perform statistical analysis.

\section{Results}

\subsection{Animal characteristics}


Body-weight related measurements and serum biochemical variables in the four experimental groups are reported in Table 1. Animals supplemented with a combination of trans-resveratrol and quercetin presented a trend towards prevention of body weight gain induced by a HFS diet intake and showed a slight reduction in liver proportions (\% body weight) (Table 1). Moreover, supplementation with trans-resveratrol and quercetin alone or in combination significantly reduced serum insulin levels. HOMA-IR was also decreased in the groups supplemented with trans-resveratrol or quercetin (Table 1). In addition, serum inflammation- and endotoxin-related markers (LBP, LPS and TNF- $\alpha$ levels) were determined. Although, slightly higher amounts were observed for LPS and TNF- $\alpha$ in trans-resveratrol supplemented rats, these results were not statistically significant (data not shown).

\subsection{Gut microbiota analysis}

At phylum level, quercetin supplementation was found to considerably modify relative percentages of detected phyla when compared to the HFS diet-fed control rats (Table 2). Quercetin supplementation was able to marginally $(p=0.053)$ reduce Firmicutes $(-34.2 \%)$. This value reflects the percentage of change measured taking into account the mean relative abundance of Firmicutes phlylum in quercetin supplemented group and the mean relative abundance of this bacterial group in the HFS diet-fed group, which is considered the reference group in the present study. Thus, these data suggest that after quercetin supplementation this bacterial phylum usually up-regulated in rats fed a HF diet [45], was reduced in a $34.2 \%$ when measured against the levels found in the reference group, although this diminution was not statistically significant after correction with the Bonferroni multiple comparison test. Moreover, a considerable decrease $(-80.5 \%)$ was detected in Firmicutes/Bacteroidetes ratio of animals supplemented with quercetin (Figure 1). In contrast, bacterial composition of faeces from the groups supplemented with trans-resveratrol or the combination of trans-resveratrol and quercetin, was not significantly affected at phylum level.

At class level, Erysipelotrichi (classified within the Firmicutes phylum), was the most affected bacterial group after quercetin supplementation, as the mean relative abundance of this bacterial group was significantly $(p=0.018)$ reduced $(-83.9 \%)$ compared to the HFS diet-fed control rats (appendix Table A.1).

At family level, groups supplemented with quercetin and the combination of trans-resveratrol and quercetin, presented a bacterial profile which substantially differed from the relative abundance of bacterial families detected in the faeces of the HFS diet-fed control and trans-resveratrol supplemented rats, being this variation especially notable for the Erysipelotrichaceae family (Figure 
2). Nevertheless, trans-resveratrol supplementation produced a statistically significant inhibition in the Graciibacteraceae family (-57.7 \%) compared to the HFS diet-fed control rats. Relative abundance (\% of total 16S rDNA) of the most representative families is also reported (Table 3).

Regarding genus level, quercetin supplementation for 6 weeks significantly reduced the levels of Bacillus genus (-74.3\%) when compared to the HFS diet-fed rats (appendix table B.1). In contrast, trans-resveratrol supplementation alone significantly lessened the abundance of Parabacteroides genus $(-76.3 \%)$ compared to the mean relative abundance found in the reference group (appendix table B.2). The combination of both polyphenols did not produce any changes at this taxonomic level.

At species level, the group supplemented with quercetin showed a statistically significant inhibition in four bacterial species after multiple adjustments with Bonferroni test (Figure 3). In this regard, it is noteworthy the statistically significant downregulation detected in the mean relative abundance of Eubacterium cylindroides (-80.4 \%) compared to the relative percentage found in the control group (Figure 4). Moreover, supplementation with trans-resveratrol significantly reduced the mean relative abundance of different Clostridia species such as Clostridium aldenense (-93.1\%), Clostridium hathewayi (-73.2 \%), Clostridium sp. C9 (-76.3 \%), Clostridium sp. MLG661 (-53.7 \%) in opposition to the mean relative abundance of Clostridium sp. XB90 (266.6 \%) that was notably enhanced when compared to the HFS diet-fed control rats (Figure 3). Furthermore, the percentage of change in the mean relative abundance of Gracilibacter thermotolerans (-57.7 \%) and Parabacteroides distasonis $(-77.4 \%)$ was negatively affected by trans-resveratrol in comparison to that detected in the HFS dietfed control group (Figure 3). As far as the effect resulted from the combined consumption of both polyphenols is concerned, the relative abundance of two Clostridia species (Clostridium clariflavum, $327.0 \%$ and Clostridium methylpentosum, $245.6 \%$ ), as well as the relative abundance of a member of Lachnospiraceae family (Blautia stercoris, $321.7 \%$ ) were significantly enhanced compared to the percentage determined in the reference group (Figure 3). Moreover, although non-statistically significant, the relative percentage of diverse bacterial species was also found to be differentially affected by the intake of polyphenols (appendix Table C.1, Table C.2 and Table C.3).

In addition, Akkermansia muciniphila ATCC BAA-835, the unique bacteria belonging to Verrucomicrobia phylum, showed a notable increase (1384.0\%) after the 6 weeks period of quercetin intake when compared to the relative abundance detected in diet-induced overweight control rats (Figure 5). However, these differences were not statistically significant. Furthermore, the relative abundance (\% of total $16 \mathrm{~S}$ rDNA) of bacterial species represented in Figure 6 , especially belonging to Bacteroidia class, such as Bacteroides sp. S-18 (105.2 \%), Bacteroides sp. dnLKV (87.8 \%), Barnesiella intestinihominis (127.6 \%), Bacteroides dorei (295.0\%), Bacteroides chinchillae (334.8\%), 
Candidatus Prevotella conceptionensis $(200.5 \%)$ were boosted by quercetin supplementation, whereas one species belonging to Deltaproteobacteria class (Bilophila wadsworthia, -36.9\%) was downregulated in comparison to the HFS diet-fed control group. These species, even if the differences were not statistically significant, were found to be the most affected after a 6 weeks dietary treatment with quercetin.

\subsection{Gene expression in colonic mucosa}

After 6 weeks of supplementation, the rats treated with quercetin showed a significantly higher colonic expression of IL-18. In contrast, the intake of trans-resveratrol produced a considerable increase in most of the measured inflammation-related parameters reaching statistical significance with TLR4, LBP and IL-18 gene expression (Figure 7). No statistical differences were found in colonic inflammation-related gene expression in the rats supplemented with the combination of both polyphenols.

Regarding variables related to intestinal permeability, those experimental groups supplemented with trans-resveratrol alone or with the mixture of trans-resveratrol and quercetin, showed a statistically significant upregulation in the expression levels of TJP-2 and Ocln gene $(p<0.05)$. In contrast, quercetin supplementation did not seem to modify the intestinal barrier permeability associated markers (Figure 8).

\subsection{SCFA analysis}

SCFA profile detected by GC/MS analysis in faecal contents of the four experimental groups revealed no statistically significant differences. Supplementation with quercetin, although not significant, was found to slightly increase concentrations of acetate $(2.00 \pm 0.24 \mathrm{mg} / \mathrm{g}$ dry weight), propionate $(0.51 \pm$ $0.07 \mathrm{mg} / \mathrm{g}$ dry weight) and butyrate $(0.58 \pm 0.11 \mathrm{mg} / \mathrm{g} \mathrm{dry}$ weight) when compared to the HFS dietfed control rats $(1.68 \pm 0.39,0.41 \pm 0.11,0.38 \pm 0.11 \mathrm{mg} / \mathrm{g}$ dry weight, respectively). In contrast, dietary supplementation with trans-resveratrol alone and with the combination of trans-resveratrol and quercetin, produced no changes in SCFA levels (appendix Table D.1).

\section{Discussion}

Therapeutic effects have been attributed to resveratrol in metabolic derangements [46], and also to quercetin, mainly due to allegedly antioxidant and anti-inflammatory properties [47, 48]. Mechanisms surrounding their beneficial effects have attracted research interest [49]. Nevertheless, 
studies investigating the interaction within bioactive compounds and gut microbes are rather limited [50-52]. The goal of the present research was to determine whether supplementation with transresveratrol and/or quercetin was able to modify gut microbiota composition, differing from microbial profile observed in HFS diet-fed control rats. Moreover, we sought to analyse whether these modifications in microbial ecology concur not only with body weight and biochemical changes, but also with alterations at intestinal epithelial level.

In the current research, dietary treatment with pure polyphenolic compounds, especially when jointly administered, decreased body weight gain. The lack of anti-obesity effect of quercetin is in agreement with data in the literature, which suggest the need of treatment periods longer than 9 weeks for this polyphenol to achieve an effective body fat lowering effect [53]. As far as resveratrol is concerned, the dose used in the present experiment was effective in reducing body fat after 6 weeks of treatment, but in a model of genetic obesity (Zucker fa/fa rats) [40,54]. In a model of obesity induced by using the same high-fat high-sucrose diet than that used in the present study, our group previously showed that a dose of $6 \mathrm{mg} / \mathrm{kg}$ bw/day resveratrol did not induce changes in adipose tissue weight [39]. By contrast, a dose of $30 \mathrm{mg} / \mathrm{kg}$ bw/day was very effective [41]. It seems that in this obesity model a dose higher than $15 \mathrm{mg} / \mathrm{kg}$ bw/day is needed to effectively reduce body fat. The dosage used in the current experiment for resveratrol and quercetin was effective to decrease insulin levels and also, to improve insulin sensitivity, these findings support results from previous studies describing resveratrol and quercetin as potential candidates for the treatment of metabolic diseases such as insulin resistance [30,31]. To our knowledge, few studies have explored the effects of pure resveratrol and quercetin consumption alone or combined, on resident microbiota composition in order to find a possible association between their beneficial properties in the host and specific gut microbial modifications [55]. In contrast, there is strong evidence supporting the role of gut microbiota in several metabolic functions [56]. Furthermore, modifications carried out in microbial ecology have been demonstrated to derive in host metabolic phenotype modulation, influencing host biochemistry and increasing host susceptibility to diseases $[57,58]$. The obese metabolic phenotype transmissibility in germ-free mice that were transplanted the gut microbiota from obese $(o b / o b)$ mice has been also proven [3]. Nevertheless, gut microbiota modifications have been attributed both, to genetic obesity [2], and to diet-induced alterations independent of obesity [45].

In general, obese gut microbiota has been repeatedly associated with a reduced Bacteroidetes/Firmicutes ratio $[2,59]$. Our findings agree with this feature as HFS diet-fed control rats exhibited the highest Firmicutes/Bacteroidetes ratio. Interestingly, quercetin administration to the HFS diet prominently decreased this ratio. Likewise, animals supplemented with this polyphenol 
showed a considerable reduction in Firmicutes phylum commonly dominating in obesity and overweight status $[14,60]$. The enrichment of Erysipelotrichaceae family in diet-induced obesity has been similarly reported in several studies [61, 62]. Indeed, a metagenomic study carried out by Turnbaugh et al. [61] in mice that developed obesity by the intake of high-fat high-sucrose diet, it was concluded that the microbiome of HFS diet-fed mice was rich in genes encoding components of a major system, which is essential for the sugar uptake for most intestinal bacteria [61]. Thus, it was suggested that Erysipelotrichaceae contributed to the function of these genes leading to a more efficient energy extraction from the diet $[61,63]$. In the current investigation, the potential of quercetin to deeply modify general bacterial profile at family level is underlined, indicating an important reduction in the relative abundance of Erysipelotrichaceae family, in contrast to what it was found with trans-resveratrol administration alone or in combination. Likewise, it was previously demonstrated by our group [17] that Erysipelotrichi taxa was profoundly enlarged (1025.4 \%) after a HFS diet treatment for 6 weeks [17]. Interestingly, concerning the enrichment found in the Bacteroidetes phylum, quercetin supplementation also deeply increased the abundance of Bacteroidaceae and Prevotellaceae families, which were stated to be negatively influenced in highfat-fed mice [45]. Besides, quercetin supplementation resulted in the reduction of the genus Bacillus, another predominant bacterium in Western diet-fed obese mice [64]. Surprisingly, trans-resveratrol solely did not produce significant modifications at the highest division levels, but was detected to follow the opposite pattern to the previously mentioned results. Furthermore, when combining both polyphenols together, gut microbiota modifications emulated quercetin's individual consequences although, these alterations were found to be weaker. Notwithstanding, at species level, transresveratrol supplementation significantly inhibited several bacterial species, such as Gracilibacter thermotolerans, Parabacteroides distasonis and species from Clostridia class (Clostridium aldenense, Clostridium hathewayi, Clostridium sp. MLG661), whereas it significantly enhanced Clostridium sp. XB90. Of especial mention, Parabacteroides distasonis has been found to be reduced in obese individuals [65] and suppressions in Clostridium aldenense and Clostridium hathewayi species, members of Clostridium cluster XIVa, have been reported to be major butyrate producers [66]. On the other hand, the differential sensitivity to polyphenols has been postulated indicating a higher sensitivity to phenolic compounds of Gram-positive bacteria rather than the Gram-negative bacteria, possibly attributable to changes in their wall composition [67]. The intake of quercetin positively affected the growth of three species (Bacteroides vulgatus, Clostridium clariflavum and Clostridium sp. MLG661) and strongly inhibited the abundance of Eubacterium cylindroides. Bacteroides vulgatus and Clostridium clariflavum were previously found to be diminished in obese subjects $[65,68]$ and HFS diet-fed overweight rats [17]. Indeed, Eubacterium cylindroides has been detected in faecal microbiota of obese humans [14], it was up-regulated in high-fat diet-induced obesity [14, 61] and 
reported to be enlarged (4072.1\%) in rats fed a HFS diet for 6 weeks [17]. Blautia stercoris, Clostridium clariflavum and Clostridium methylpentosum were also up-regulated by the combination of polyphenols. In agreement with these results, Clostridium and Eubacterium have been considered as the main genera involved in the metabolism of a large variety of phenolic compounds, including isoflavones (daidzein), flavones (naringenin and ixoxanthumol), flavan-3-ols (catechin and epicatechin) and flavonols (quercetin and kaempferol) [69]. According to our data, given the poor absorption of quercetin into the bloodstream from the gut, the mechanisms contributing to the amelioration of insulin resistance might imply a direct gut microbiota modulatory effect. In contrast, resveratrol might act as an anti-microbial compound that counteracts the individual effects of quercetin, suggesting that the improvements in insulin resistance could be due to indirect mechanisms which do not involve big changes in gut microbiota composition.

Given the connection between gut microbiota, metabolism and intestinal epithelium [70], the expression of genes implicated in the permeability of the gut epithelium was analysed. It is widely known that intact mucosal barrier is essential to avoid the development of inflammation [71]. Indeed, impaired intestinal barrier function is regarded as a relevant contributory factor to obesity and associated metabolic disturbances $[4,72]$. In the present study, although being aware that the fasting period to which animals were subjected before sacrifice might have an effect on intestinal integrity [73], an upregulation in the expression of TJPs and Ocln was found to be induced by the administration of trans-resveratrol alone and when combined with quercetin, which was not perceived with the intake of quercetin alone. Furthermore, the expression levels of inflammationassociated genes were induced by trans-resveratrol supplementation such as, the expression of TLR2 and specially, TLR4, LBP and IL-18. TLRs, more specifically TLR2 and TLR4, have been largely implicated in inflammatory states in the gastrointestinal tract [74-76]. TLRs comprise a family of pattern-recognition receptors that identify conserved molecular products of microorganisms, such as lipopolysaccharides and lipoteichoic acid (LTA) [77]. Thus, TLR2 recognizes Gram-positive bacteria derived products, whereas TLR4 is stimulated by LPS derived from Gram-negative bacteria [77]. Their expression is dependent on dysbiosis associated to altered host-bacterial interactions [78]. Indeed, TLRs act as sensors of microbial infection, initiating inflammatory and immune defence responses [79]. However, the bacterial ligands are shared by all classes of bacteria, being also produced by commensal microorganisms [80]. In line with this statement, Rakoff-Nahoum et al. demonstrated a non-immune function of TLRs in the maintenance of epithelial homeostasis [80]. Based on the recognition of commensal bacteria as health- promoting "symbionts", emerging evidence has confirmed the role of luminal microbes to signal the interfacing epithelial layer, to control the turnover rate of enterocytes and to fortify the epithelial turnover regenerative and barrier functions 
[81]. Rakoff-Nahoum et al. suggested a beneficial role generated from the recognition of commensal bacteria by TLRs [80]. The recognition of commensal bacteria and posterior activation of TLRs was hypothesized to induce generation of protective factors (i.e. cytokines) upon epithelial damage that have been previously reported in vivo and in vitro to play a beneficial role by initiating repair responses (i.e. process known as restitution) [82] and have been defined as markers of protective responses in colonic tissue [80]. In fact, a growing body of evidence indicates the relevance of certain mediators (IL- $\alpha, \mathrm{IL}-1 \beta, \mathrm{IL}-18$ ) to preserve mucosal homeostasis, as well as to protect and restore epithelial barrier [83]. In particular, the up-regulation of IL-18 (a family member of IL-1) found in our study might be related with its dichotomous role in the gut, as IL-18 derived from intestinal epithelial cells has been described to facilitate tissue repair and promote mechanisms to induce homeostasis in early phase of inflammation [84]. Experiments conducted by other researchers have concluded the essential role of IL-18 to maintain epithelial integrity and prevent the translocation of bacteria, associating this feature to the activation of TLRs in inducing the signal to repair $[85,86]$. From our data, a presumable promotion of intestinal epithelial repair by protective factors (i.e. IL-18) induced by commensal bacteria-derived activation of TLR signalling might be hypothesized, particularly after trans-resveratrol administration as a possible mechanism leading to intestinal epithelial homeostasis. It might be speculated that although non-statistically significant, inflammation- and endotoxinrelated markers (LBP, TLR4, TLR2 expression levels, LPS serum levels) showed a trend to a subtle increase after trans-resveratrol supplementation. This outcome might underlie a slight inflammation and immune response taking place in the intestine [87]. TLRs could be acting upon damage as a defence mechanism to prevent bacteria and bacterial products translocation through rearrangement of tight-junction proteins to enhance intestinal epithelial integrity [88-90]. Nevertheless, these results deserve further research in order to confirm the mechanisms through which polyphenols interact with gut microbiota to cause host gene expression modifications, and also to be able to conclude the overall health consequences of this interactions in health.

\section{Conclusions}

From this experiment, it was concluded that the intake of trans-resveratrol and quercetin at the same time acted synergistically to reduce body-weight gain. Moreover, administration of both polyphenols alone or in combination improved basal insulin levels and HOMA-IR index. On the other hand, one of the major findings was related to the impact of quercetin supplementation on gut microbiota composition. In fact, treatment with quercetin attenuated the increase in Firmicutes/Bacteroidetes ratio in HFS diet-fed rats and notably modified gut microbial pattern at family level, significantly reducing the abundance of Erysipelotrichaceae and the genus Bacillus, 
closely related to the consumption of western diets and body weight gain. Furthermore, supplementation with quercetin produced a significant alteration in specific bacterial species, rising some that have been inversely related to obesity (Bacteroides vulgatus, Akkermansia muciniphila) and reducing the relative abundance of others associated to diet-induced obesity (Eubacterium cylindroides, Bilophila wadsworthia). In contrast, after trans-resveratrol supplementation no profound effects on gut microbiota composition were identified, especially at the highest division levels. Indeed, from results combining trans-resveratrol with quercetin, it can be speculated that individual effects attributed to quercetin were lessened. On the other hand, trans-resveratrol supplementation altered the mRNA expression levels of TJPs as well as some inflammation associated markers.

In summary, this study demonstrates the gut microbiota modulatory role of quercetin when administered to HFS diet-fed overweight animals, and left some doubts for future research about the mechanisms of trans-resveratrol to exert health benefits due to its uncertain potential to modulate gut microbial population and its impact on host intestinal gene expression. Limitations of this study are that there is no a group fed with a standard diet to identify the modifications induced by the type of diet to conclude whether polyphenols, especially quercetin, reversed diet-induced gut microbiota dysbiosis. On the other hand, this study could not differentiate whether the outcomes discovered were derived from the original compounds provided or from the metabolites produced in the gut. Another issue to be considered is the fact that SCFA measurements were conducted ex-vivo in faeces, which could not be an estimation of the real production of SCFA, as rats are caecum fermenters and a great amount of these compounds (95-99\%) are absorbed in the large intestine [91]. In addition, the measurement of the mRNA levels of TJPs is an indirect method to assess the impact of these polyphenols on gut integrity; gene expression levels are not always a reflection of their functional active proteins. As a result, we should be cautious as further analyses are required to be able to draw conclusions about the protective functions of these polyphenols on intestinal epithelial barrier. Furthermore, an important question addressed in this study is that the positive effects derived from the combination of trans-resveratrol and quercetin in body weight, were not explained by gut microbiota modifications. Quercetin produced the most profound effect on gut microbial ecology, and this outcome was associated with significant metabolic improvements. In contrast, the administration of trans-resveratrol also improved some metabolic derangements, but this effect was not mediated by a modulatory effect on gut microbiota. From the present data, it could be stated that quercetin supplementation, robustly reshapes gut microbiota dysbiosis associated to a westernized dietary intake, which presumably might contribute to beneficial metabolic effects. Besides, from the interaction between polyphenols and bacteria, changes at 
intestinal level might be postulated in the host (local inflammatory tone and intestinal mucosal barrier integrity), principally with trans-resveratrol administration.

\section{Acknowledgments}

This study was supported by grants from the Ministerio de Economía y Competitividad (AGL201127406-ALI), Instituto de Salud Carlos III (CIBERobn), Government of the Basque Country (IT-572-13) and University of the Basque Country (UPV/EHU) (ELDUNANOTEK UFI11/32). The authors wish to acknowledge Línea Especial about Nutrition, Obesity and Health (University of Navarra LE/97, Spain) for the financial support and the Department of Education, Language policy and Culture from Government of the Basque Country for the predoctoral grant given to Usune Etxeberria.

\section{Abbreviations}

HFS, high- fat sucrose; TJPs, tight-junction proteins; HOMA-IR, homeostasis model assessment of insulin resistance; LPS, lipopolysaccharide; LBP, LPS- binding protein; MIDs, multiplex identifiers; OTU, operational taxonomic unit; IL-18, interleukin- 18; NFk $\beta$, nuclear factor kappa $\beta$; TNF- $\alpha$, tumor necrosis factor $\alpha$; TLR-2, toll- like receptor 2; TLR-4, toll- like receptor 4; MyD88, Myeloid differentiation primary response 88; NKG2D or KIrk1, Killer cell lectin- like receptor subfamily K, member 1; TJP-1, tight-junction protein 1; TJP-2, tight-junction protein 2; Ocln, occludin; GAPDH, glyceraldehyde-3- phosphate dehydrogenase; SCFA, short-chain fatty acid; GC/MS, gaschromatography-mass spectrometry; $\mathrm{PCl}$, positive chemical ionization; SEM, standard error of the mean; LTA, lipoteichoic acid. 


\section{References}

[1] Finucane MM, Stevens GA, Cowan MJ, Danaei G, Lin JK, Paciorek CJ, et al. National, regional, and global trends in body-mass index since 1980: systematic analysis of health examination surveys and epidemiological studies with 960 country-years and 9.1 million participants. Lancet 2011; 377:55767.

[2] Ley RE, Backhed F, Turnbaugh P, Lozupone CA, Knight RD, Gordon JI. Obesity alters gut microbial ecology. Proc Natl Acad Sci U S A 2005; 102:11070-5.

[3] Turnbaugh PJ, Ley RE, Mahowald MA, Magrini V, Mardis ER, Gordon JI. An obesity-associated gut microbiome with increased capacity for energy harvest. Nature 2006; 444:1027-31.

[4] Cani PD, Bibiloni R, Knauf C, Waget A, Neyrinck AM, Delzenne NM, et al. Changes in gut microbiota control metabolic endotoxemia-induced inflammation in high-fat diet-induced obesity and diabetes in mice. Diabetes 2008; 57:1470-81.

[5] Yin YN, Yu QF, Fu N, Liu XW, Lu FG. Effects of four Bifidobacteria on obesity in high-fat diet induced rats. World J Gastroenterol 2010; 16:3394-401.

[6] Brun P, Castagliuolo I, Di Leo V, Buda A, Pinzani M, Palu G, et al. Increased intestinal permeability in obese mice: new evidence in the pathogenesis of nonalcoholic steatohepatitis. Am J Physiol Gastrointest Liver Physiol 2007; 292:G518-25.

[7] Shen J, Obin MS, Zhao L. The gut microbiota, obesity and insulin resistance. Mol Aspects Med 2013; 34:39-58.

[8] Tlaskalova-Hogenova H, Stepankova R, Kozakova H, Hudcovic T, Vannucci L, Tuckova L, et al. The role of gut microbiota (commensal bacteria) and the mucosal barrier in the pathogenesis of inflammatory and autoimmune diseases and cancer: contribution of germ-free and gnotobiotic animal models of human diseases. Cell Mol Immunol 2011; 8:110-20.

[9] Power SE, O'Toole PW, Stanton C, Ross RP, Fitzgerald GF. Intestinal microbiota, diet and health. Br J Nutr 2014; 111:387-402.

[10] Serino M, Chabo C, Burcelin R. Intestinal MicrobiOMICS to define health and disease in human and mice. Curr Pharm Biotechnol 2012; 13:746-58.

[11] Kellermayer R, Dowd SE, Harris RA, Balasa A, Schaible TD, Wolcott RD, et al. Colonic mucosal DNA methylation, immune response, and microbiome patterns in Toll-like receptor 2-knockout mice. FASEB J 2011; 25:1449-60.

[12] Vital M, Howe AC, Tiedje JM. Revealing the bacterial butyrate synthesis pathways by analyzing (meta)genomic data. MBio 2014; 5:e00889.

[13] Khanna S, Tosh PK. A clinician's primer on the role of the microbiome in human health and disease. Mayo Clin Proc 2014; 89:107-14.

[14] Ley RE, Turnbaugh PJ, Klein S, Gordon JI. Microbial ecology: human gut microbes associated with obesity. Nature 2006; 444:1022-3. 
[15] Duncan SH, Lobley GE, Holtrop G, Ince J, Johnstone AM, Louis P, et al. Human colonic microbiota associated with diet, obesity and weight loss. Int J Obes (Lond) 2008; 32:1720-4.

[16] Zhang H, DiBaise JK, Zuccolo A, Kudrna D, Braidotti M, Yu Y, et al. Human gut microbiota in obesity and after gastric bypass. Proc Natl Acad Sci U S A 2009; 106:2365-70.

[17] Etxeberria U, Arias N, Boque N, Macarulla MT, Portillo MP, Milagro Fl, et al. Shifts in microbiota species and fermentation products in a dietary model enriched in fat and sucrose. Benef Microbes 2014; 11:1-15.

[18] Karlsson F, Tremaroli V, Nielsen J, Backhed F. Assessing the human gut microbiota in metabolic diseases. Diabetes 2013; 62:3341-9.

[19] Delzenne NM, Neyrinck AM, Backhed F, Cani PD. Targeting gut microbiota in obesity: effects of prebiotics and probiotics. Nat Rev Endocrinol 2011; 7:639-46.

[20] Xu X, Xu P, Ma C, Tang J, Zhang X. Gut microbiota, host health, and polysaccharides. Biotechnol Adv 2013; 31:318-37.

[21] Delzenne NM, Neyrinck AM, Cani PD. Modulation of the gut microbiota by nutrients with prebiotic properties: consequences for host health in the context of obesity and metabolic syndrome. Microb Cell Fact 2011; 10 Suppl 1:S10.

[22] Ali F, Ismail A, Kersten S. Molecular mechanisms underlying the potential antiobesity-related diseases effect of cocoa polyphenols. Mol Nutr Food Res 2014; 58:33-48.

[23] de la Iglesia R, Milagro $\mathrm{FI}$, Campion J, Boque N, Martinez JA. Healthy properties of proanthocyanidins. Biofactors 2010; 36:159-68.

[24] de la Garza AL, Milagro FI, Boque N, Campion J, Martinez JA. Natural inhibitors of pancreatic lipase as new players in obesity treatment. Planta Med 2011; 77:773-85.

[25] Babu PV, Liu D, Gilbert ER. Recent advances in understanding the anti-diabetic actions of dietary flavonoids. J Nutr Biochem 2013; 24:1777-89.

[26] Etxeberria U, de la Garza AL, Campion J, Martinez JA, Milagro Fl. Antidiabetic effects of natural plant extracts via inhibition of carbohydrate hydrolysis enzymes with emphasis on pancreatic alpha amylase. Expert Opin Ther Targets 2012; 16:269-97.

[27] Zhang M, Swarts SG, Yin L, Liu C, Tian Y, Cao Y, et al. Antioxidant properties of quercetin. Adv Exp Med Biol 2011; 701:283-9.

[28] Rivera L, Moron R, Sanchez M, Zarzuelo A, Galisteo M. Quercetin ameliorates metabolic syndrome and improves the inflammatory status in obese Zucker rats. Obesity (Silver Spring) 2008; 16:2081-7.

[29] Poulsen MM, Jorgensen JO, Jessen N, Richelsen B, Pedersen SB. Resveratrol in metabolic health: an overview of the current evidence and perspectives. Ann N Y Acad Sci 2013; 1290:74-82.

[30] Henagan TM, Lenard NR, Gettys TW, Stewart LK. Dietary quercetin supplementation in mice increases skeletal muscle PGC1alpha expression, improves mitochondrial function and attenuates insulin resistance in a time-specific manner. PLoS One 2014; 9:e89365. 
[31] Liu K, Zhou R, Wang B, Mi MT. Effect of resveratrol on glucose control and insulin sensitivity: a meta-analysis of 11 randomized controlled trials. Am J Clin Nutr 2014; 99:1510-9.

[32] Del Rio D, Rodriguez-Mateos A, Spencer JP, Tognolini M, Borges G, Crozier A. Dietary (poly)phenolics in human health: structures, bioavailability, and evidence of protective effects against chronic diseases. Antioxid Redox Signal 2013; 18:1818-92.

[33] Cardona F, Andres-Lacueva C, Tulipani S, Tinahones FJ, Queipo-Ortuno MI. Benefits of polyphenols on gut microbiota and implications in human health. J Nutr Biochem 2013; 24:1415-22.

[34] Walle T, Hsieh F, DeLegge MH, Oatis JE, Jr., Walle UK. High absorption but very low bioavailability of oral resveratrol in humans. Drug Metab Dispos 2004; 32:1377-82.

[35] Wang D, Hang T, Wu C, Liu W. Identification of the major metabolites of resveratrol in rat urine by HPLC-MS/MS. J Chromatogr B Analyt Technol Biomed Life Sci 2005; 829:97-106.

[36] Burkon A, Somoza V. Quantification of free and protein-bound trans-resveratrol metabolites and identification of trans-resveratrol-C/O-conjugated diglucuronides - two novel resveratrol metabolites in human plasma. Mol Nutr Food Res 2008; 52:549-57.

[37] Bode LM, Bunzel D, Huch M, Cho GS, Ruhland D, Bunzel M, et al. In vivo and in vitro metabolism of trans-resveratrol by human gut microbiota. Am J Clin Nutr 2013; 97:295-309.

[38] Etxeberria U, Fernandez-Quintela A, Milagro FI, Aguirre L, Martinez JA, Portillo MP. Impact of polyphenols and polyphenol-rich dietary sources on gut microbiota composition. J Agric Food Chem 2013; 61:9517-33.

[39] Macarulla MT, Alberdi G, Gomez S, Tueros I, Bald C, Rodriguez VM, et al. Effects of different doses of resveratrol on body fat and serum parameters in rats fed a hypercaloric diet. J Physiol Biochem 2009; 65:369-76.

[40] Gomez-Zorita S, Fernandez-Quintela A, Macarulla MT, Aguirre L, Hijona E, Bujanda L, et al. Resveratrol attenuates steatosis in obese Zucker rats by decreasing fatty acid availability and reducing oxidative stress. Br J Nutr 2012; 107:202-10.

[41] Alberdi G, Rodriguez VM, Miranda J, Macarulla MT, Arias N, Andres-Lacueva C, et al. Changes in white adipose tissue metabolism induced by resveratrol in rats. Nutr Metab (Lond) 2011; 8:29.

[42] Matthews DR, Hosker JP, Rudenski AS, Naylor BA, Treacher DF, Turner RC. Homeostasis model assessment: insulin resistance and beta-cell function from fasting plasma glucose and insulin concentrations in man. Diabetologia 1985; 28:412-9.

[43] Chevreux B, Pfisterer T, Drescher B, Driesel AJ, Muller WE, Wetter T, et al. Using the miraEST assembler for reliable and automated mRNA transcript assembly and SNP detection in sequenced ESTs. Genome Res 2004; 14:1147-59.

[44] Huson DH, Mitra S, Ruscheweyh HJ, Weber N, Schuster SC. Integrative analysis of environmental sequences using MEGAN4. Genome Res 2011; 21:1552-60.

[45] Hildebrandt MA, Hoffmann C, Sherrill-Mix SA, Keilbaugh SA, Hamady M, Chen YY, et al. High-fat diet determines the composition of the murine gut microbiome independently of obesity. Gastroenterology 2009; 137:1716-24 e1-2. 
[46] Jeon BT, Jeong EA, Shin HJ, Lee Y, Lee DH, Kim HJ, et al. Resveratrol attenuates obesityassociated peripheral and central inflammation and improves memory deficit in mice fed a high-fat diet. Diabetes 2012; 61:1444-54.

[47] Timmers S, Hesselink MK, Schrauwen P. Therapeutic potential of resveratrol in obesity and type 2 diabetes: new avenues for health benefits? Ann N Y Acad Sci 2013; 1290:83-9.

[48] Boots AW, Haenen GR, Bast A. Health effects of quercetin: from antioxidant to nutraceutical. Eur J Pharmacol 2008; 585:325-37.

[49] Farghali H, Kutinova Canova N, Lekic N. Resveratrol and related compounds as antioxidants with an allosteric mechanism of action in epigenetic drug targets. Physiol Res 2013; 62:1-13.

[50] van Duynhoven J, Vaughan EE, van Dorsten F, Gomez-Roldan V, de Vos R, Vervoort J, et al. Interactions of black tea polyphenols with human gut microbiota: implications for gut and cardiovascular health. Am J Clin Nutr 2013; 98:1631S-41S.

[51] Queipo-Ortuno MI, Boto-Ordonez M, Murri M, Gomez-Zumaquero JM, Clemente-Postigo M, Estruch R, et al. Influence of red wine polyphenols and ethanol on the gut microbiota ecology and biochemical biomarkers. Am J Clin Nutr 2012; 95:1323-34.

[52] Sanchez-Patan F, Cueva C, Monagas M, Walton GE, Gibson GR, Quintanilla-Lopez JE, et al. In vitro fermentation of a red wine extract by human gut microbiota: changes in microbial groups and formation of phenolic metabolites. J Agric Food Chem 2012; 60:2136-47.

[53] Arias N, Macarulla MT, Aguirre L, Martinez-Castano MG, Portillo MP. Quercetin can reduce insulin resistance without decreasing adipose tissue and skeletal muscle fat accumulation. Genes Nutr 2014; 9:361. Doi: 10.1007/s12263-013-0361-7

[54] Gomez-Zorita S, Fernandez-Quintela A, Lasa A, Hijona E, Bujanda L, Portillo MP. Effects of resveratrol on obesity-related inflammation markers in adipose tissue of genetically obese rats. Nutrition 2013; 29:1374-80.

[55] Qiao Y, Sun J, Xia S, Tang X, Shi Y, Le G. Effects of resveratrol on gut microbiota and fat storage in a mouse model with high-fat-induced obesity. Food Funct 2014; 5:1241-9.

[56] Blaut M, Clavel T. Metabolic Diversity of the Intestinal Microbiota: Implications for Health and Disease. The Journal of Nutrition 2007; 137:751S-5S.

[57] Nicholson JK, Wilson ID. Opinion: understanding 'global' systems biology: metabonomics and the continuum of metabolism. Nat Rev Drug Discov 2003; 2:668-76.

[58] Nicholson JK, Holmes E, Kinross J, Burcelin R, Gibson G, Jia W, et al. Host-Gut Microbiota Metabolic Interactions. Science 2012; 336:1262-7.

[59] Duca FA, Sakar Y, Lepage P, Devime F, Langelier B, Dore J, et al. Replication of obesity and associated signaling pathways through transfer of microbiota from obese-prone rats. Diabetes 2014; 63:1624-36.

[60] Turnbaugh PJ, Hamady M, Yatsunenko T, Cantarel BL, Duncan A, Ley RE, et al. A core gut microbiome in obese and lean twins. Nature 2009; 457:480-4. 
[61] Turnbaugh PJ, Backhed F, Fulton L, Gordon JI. Diet-induced obesity is linked to marked but reversible alterations in the mouse distal gut microbiome. Cell Host Microbe 2008; 3:213-23.

[62] Fleissner CK, Huebel N, Abd El-Bary MM, Loh G, Klaus S, Blaut M. Absence of intestinal microbiota does not protect mice from diet-induced obesity. Br J Nutr 2010; 104:919-29.

[63] Blaut M, Klaus S. Intestinal Microbiota and Obesity. In: Joost H-G, editor. Appetite Control: Springer Berlin Heidelberg; 2012. p. 251-73.

[64] Turnbaugh PJ, Ridaura VK, Faith JJ, Rey FE, Knight R, Gordon JI. The effect of diet on the human gut microbiome: a metagenomic analysis in humanized gnotobiotic mice. Sci Transl Med 2009; 1:6ra14.

[65] Verdam FJ, Fuentes S, de Jonge C, Zoetendal EG, Erbil R, Greve JW, et al. Human intestinal microbiota composition is associated with local and systemic inflammation in obesity. Obesity (Silver Spring) 2013; 21:E607-15.

[66] Neyrinck AM, Possemiers S, Verstraete W, De Backer F, Cani PD, Delzenne NM. Dietary modulation of clostridial cluster XIVa gut bacteria (Roseburia spp.) by chitin-glucan fiber improves host metabolic alterations induced by high-fat diet in mice. J Nutr Biochem 2012; 23:51-9.

[67] Puupponen-Pimia R, Nohynek L, Hartmann-Schmidlin S, Kahkonen M, Heinonen M, MaattaRiihinen $\mathrm{K}$, et al. Berry phenolics selectively inhibit the growth of intestinal pathogens. J Appl Microbiol 2005; 98:991-1000.

[68] Bervoets L, Van Hoorenbeeck K, Kortleven I, Van Noten C, Hens N, Vael C, et al. Differences in gut microbiota composition between obese and lean children: a cross-sectional study. Gut Pathog 2013; 5:10. Doi: 10.1186/1757-4749-5-10

[69] Selma MV, Espin JC, Tomas-Barberan FA. Interaction between phenolics and gut microbiota: role in human health. J Agric Food Chem 2009; 57:6485-501.

[70] Tremaroli $V$, Backhed F. Functional interactions between the gut microbiota and host metabolism. Nature 2012; 489:242-9.

[71] Hooper LV, Wong MH, Thelin A, Hansson L, Falk PG, Gordon JI. Molecular analysis of commensal host-microbial relationships in the intestine. Science 2001; 291:881-4.

[72] Teixeira TF, Collado MC, Ferreira CL, Bressan J, Peluzio Mdo C. Potential mechanisms for the emerging link between obesity and increased intestinal permeability. Nutr Res 2012; 32:637-47.

[73] Yamauchi K, Kamisoyama H, Isshiki Y. Effects of fasting and refeeding on structures of the intestinal villi and epithelial cells in White Leghorn hens. Br Poult Sci 1996; 37:909-21.

[74] Erridge C, Duncan SH, Bereswill S, Heimesaat MM. The induction of colitis and ileitis in mice is associated with marked increases in intestinal concentrations of stimulants of TLRs 2, 4, and 5. PLoS One 2010; 5:e9125.

[75] Buchholz BM, Bauer AJ. Membrane TLR signaling mechanisms in the gastrointestinal tract during sepsis. Neurogastroenterol Motil 2010; 22:232-45. 
[76] Teran-Ventura E, Aguilera M, Vergara P, Martinez V. Specific changes of gut commensal microbiota and TLRs during indomethacin-induced acute intestinal inflammation in rats. J Crohns Colitis 2014; 8:1043-54.

[77] Takeda K, Kaisho T, Akira S. Toll-like receptors. Annu Rev Immunol 2003; 21:335-76.

[78] Tanaka K. Expression of Toll-like receptors in the intestinal mucosa of patients with inflammatory bowel disease. Expert Rev Gastroenterol Hepatol 2008; 2:193-6.

[79] Ewaschuk JB, Backer JL, Churchill TA, Obermeier F, Krause DO, Madsen KL. Surface expression of Toll-like receptor 9 is upregulated on intestinal epithelial cells in response to pathogenic bacterial DNA. Infect Immun 2007; 75:2572-9.

[80] Rakoff-Nahoum S, Paglino J, Eslami-Varzaneh F, Edberg S, Medzhitov R. Recognition of commensal microflora by toll-like receptors is required for intestinal homeostasis. Cell 2004; 118:229-41.

[81] Yu LC, Wang JT, Wei SC, Ni YH. Host-microbial interactions and regulation of intestinal epithelial barrier function: From physiology to pathology. World J Gastrointest Pathophysiol 2012; 3:27-43.

[82] Yoo J LML, Matthews J.B. Cytokines in restitution. Baltimore, Md.: Karger; 2002.

[83] Lopetuso LR, Chowdhry S, Pizarro TT. Opposing Functions of Classic and Novel IL-1 Family Members in Gut Health and Disease. Front Immunol 2013; 4:181.

[84] Reuter BK, Pizarro TT. Commentary: the role of the IL-18 system and other members of the IL$1 R / T L R$ superfamily in innate mucosal immunity and the pathogenesis of inflammatory bowel disease: friend or foe? Eur J Immunol 2004; 34:2347-55.

[85] Dupaul-Chicoine J, Yeretssian G, Doiron K, Bergstrom KS, McIntire CR, LeBlanc PM, et al. Control of intestinal homeostasis, colitis, and colitis-associated colorectal cancer by the inflammatory caspases. Immunity 2010; 32:367-78.

[86] Siegmund B. Interleukin-18 in intestinal inflammation: friend and foe? Immunity 2010; 32:300-2.

[87] Fasano A, Shea-Donohue T. Mechanisms of disease: the role of intestinal barrier function in the pathogenesis of gastrointestinal autoimmune diseases. Nat Clin Pract Gastroenterol Hepatol 2005; 2:416-22.

[88] Hsu D, Fukata M, Hernandez YG, Sotolongo JP, Goo T, Maki J, et al. Toll-like receptor 4 differentially regulates epidermal growth factor-related growth factors in response to intestinal mucosal injury. Lab Invest 2010; 90:1295-305.

[89] Cario E, Gerken G, Podolsky DK. Toll-like receptor 2 enhances ZO-1-associated intestinal epithelial barrier integrity via protein kinase C. Gastroenterology 2004; 127:224-38.

[90] Cario E, Gerken G, Podolsky DK. Toll-like receptor 2 controls mucosal inflammation by regulating epithelial barrier function. Gastroenterology 2007; 132:1359-74.

[91] Campbell JM, Fahey GC, Jr., Wolf BW. Selected indigestible oligosaccharides affect large bowel mass, cecal and fecal short-chain fatty acids, pH and microflora in rats. J Nutr 1997; 127:130-6. 


\section{TABLE LEGENDS}

1. Table 1. Body weight-related measurements and serum biochemical parameters of animals fed with a HFS diet supplemented or not with trans-resveratrol and quercetin for 6 weeks.

All results are expressed as the mean \pm SEM. Statistical analyses were performed using One-Way ANOVA followed by Dunnett post-hoc test. Data with different superscript letters are significantly different, $p<0.05$. HFS, high- fat sucrose diet-fed control rats; RSV, supplemented with $15 \mathrm{mg} / \mathrm{kg} \mathrm{BW/day} \mathrm{of} \mathrm{trans-resveratrol;}$ $\mathrm{Q}$, supplemented with $30 \mathrm{mg} / \mathrm{kg} \mathrm{BW/day}$ of quercetin; RSV $+\mathrm{Q}$, supplemented with a combination of transresveratrol and quercetin at the same doses.

2.

able 2. Relative abundance (\% of total $16 \mathrm{~S}$ rDNA) of detected bacterial phyla in faeces of HFS diet- fed rats supplemented or not with trans-resveratrol and quercetin for 6 weeks.

All results are expressed as the mean \pm SEM. Statistical analyses were performed using Kruskal-Wallis followed by Mann Whitney $U$ test and $p$ values were corrected with the Bonferroni test. $t<0.1$ after correction with the Bonferroni multiple comparison test was set out as marginal trend. NS, non-significant values after Kruskal-Wallis comparison test. HFS, high-fat sucrose diet-fed control rats; RSV, supplemented

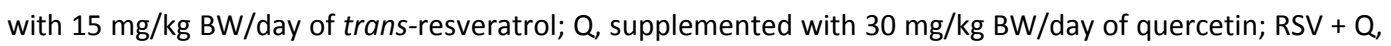
supplemented with a combination of trans-resveratrol and quercetin at the same doses.

3.

able 3. Relative abundance (\% of total $16 \mathrm{~S}$ rDNA) of the most representative families in faeces of HFS diet-fed rats supplemented or not with trans-resveratrol and quercetin for 6 weeks.

Results are expressed as the mean \pm SEM of the relative abundance (\% of total $16 \mathrm{~S}$ rDNA) of different families detected in faecal samples by $16 \mathrm{~S}$ rDNA pyrosequencing. Statistical analyses were performed using KruskalWallis followed by Mann Whitney $U$ test and $p$ values were corrected by Bonferroni test, considering $p<0.05$ statistically significant after correction with the Bonferroni multiple comparison test. Data with different superscript letters are significantly different. NS, non- significant values after Kruskal-Wallis comparison test. HFS, high-fat sucrose diet; RSV, supplemented with trans-resveratrol $15 \mathrm{mg} / \mathrm{kg} \mathrm{BW/} \mathrm{day;} \mathrm{Q,} \mathrm{supplemented} \mathrm{with}$ quercetin $30 \mathrm{mg} / \mathrm{kg} \mathrm{BW/day;} \mathrm{RSV+Q}$, supplemented with a combination of trans-resveratrol + quercetin at the same doses.

\section{FIGURE LEGENDS}

1. Figure 1. Firmicutes/Bacteroidetes ratio of HFS diet-fed rats supplemented or not with trans-resveratrol and quercetin for 6 weeks. Results are expressed as mean \pm SEM of the relative abundance (\% of total 16 S rDNA) of each phylum. HFS, high-fat sucrose diet-fed control rats; RSV, supplemented with $15 \mathrm{mg} / \mathrm{kg}$ BW/day of transresveratrol; $Q$, supplemented with $30 \mathrm{mg} / \mathrm{kg} B W /$ day of quercetin; RSV $+Q$, supplemented with a combination of trans-resveratrol and quercetin at the same doses.

2. Figure 2. Relative bacterial composition at family level (\% of total $16 \mathrm{~S}$ rDNA) in faeces of HFS diet-fed rats supplemented or not with trans-resveratrol and quercetin for 6 weeks. All data are displayed on a relative scale based on the 16S rDNA frequency taxa. HFS, high-fat sucrose diet; RSV, supplemented with trans-resveratrol 15 $\mathrm{mg} / \mathrm{kg} \mathrm{BW} /$ day; $\mathrm{Q}$, supplemented with quercetin $30 \mathrm{mg} / \mathrm{kg} B W /$ day; $R S V+Q$, supplemented with a combination of trans-resveratrol + quercetin at the same doses.

3. Figure 3. Relative abundance (\% of total $16 \mathrm{~S}$ rDNA) of bacterial species significantly different from the HFS diet-fed control rats. Results are expressed as the mean \pm SEM. Statistical analyses were performed using Kruskal-Wallis followed by Mann Whitney $U$ test and $p$ values were corrected with the Bonferroni test. ${ }^{*} p<0.05$ 
after correction with the Bonferroni multiple comparison test. NS, non- significant values after Kruskal-Wallis comparison test. ${ }^{*} p<0.05$, HFS vs RSV; ${ }^{\$} p<0.05$ HFS vs $Q ;{ }^{*} p<0.05$, HFS vs RSV+Q. HFS, high-fat sucrose diet; RSV, supplemented with trans-resveratrol $15 \mathrm{mg} / \mathrm{kg} B W /$ day; $Q$, supplemented with quercetin $30 \mathrm{mg} / \mathrm{kg}$ $\mathrm{BW} /$ day; RSV+Q, supplemented with a combination of trans-resveratrol + quercetin at the same doses.

4. Figure 4. Relative abundance (\% of total $16 \mathrm{~S}$ rDNA) of Eubacterium cylindroides in faeces of HFS diet-fed rats supplemented or not with trans-resveratrol and quercetin for 6 weeks. Results are expressed as the mean \pm SEM. Statistical analyses were performed using Kruskal-Wallis followed by Mann Whitney $U$ test and $p$ values were corrected with the Bonferroni test. ${ }^{\$} p<0.05$, HFS vs $Q$ after correction with the Bonferroni multiple comparison test. HFS, high-fat sucrose diet; RSV, supplemented with trans-resveratrol $15 \mathrm{mg} / \mathrm{kg} \mathrm{BW/} \mathrm{day;} \mathrm{Q,}$

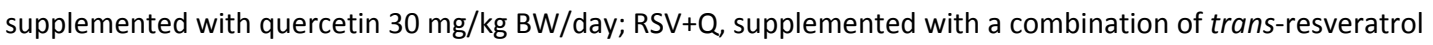
+ quercetin at the same doses.

5. Figure 5. Relative abundance (\% of total $16 \mathrm{~S}$ rDNA) of Akkermansia muciniphila ATCC BAA-835 in faeces of HFS diet-fed control rats supplemented or not with trans-resveratrol and quercetin for $\mathbf{6}$ weeks. Results are expressed as the mean \pm SEM. Statistical analyses were performed using Kruskal-Wallis followed by Mann Whitney $U$ test and $p$ values were corrected with the Bonferroni test. HFS, high-fat sucrose diet; RSV, supplemented with trans-resveratrol $15 \mathrm{mg} / \mathrm{kg} \mathrm{BW/} \mathrm{day;} \mathrm{Q}$, supplemented with quercetin $30 \mathrm{mg} / \mathrm{kg} \mathrm{BW/day;}$ $\mathrm{RSV}+\mathrm{Q}$, supplemented with a combination of trans-resveratrol + quercetin at the same doses.

6. Figure 6. Relative abundance (\% of total $16 \mathrm{~S}$ rDNA) of bacterial species in faeces of HFS diet-fed control rats supplemented or not with trans-resveratrol and quercetin for 6 weeks. Results are expressed as the mean \pm SEM. HFS, high-fat sucrose diet; RSV, supplemented with trans-resveratrol $15 \mathrm{mg} / \mathrm{kg} \mathrm{BW} /$ day; $Q$, supplemented with quercetin $30 \mathrm{mg} / \mathrm{kg} \mathrm{BW} /$ day; RSV $+Q$, supplemented with a combination of trans-resveratrol + quercetin at the same doses.

7. Figure 7. Relative mRNA expression of inflammation-related genes in colonic mucosa. Results are expressed as the mean \pm SEM. Statistical analyses were performed using Kruskal-Wallis followed by Mann Whitney $U$ test. $^{*} p<0.05$, HFS vs RSV; $\$ p<0.05$, HFS vs $Q$. TNF $\alpha$, tumor necrosis factor $\alpha$; TLR2, Toll-like receptor 2; TLR4, Toll-like receptor 4; LBP, Lipopolysaccharide binding protein; NFK $\beta$, Nuclear factor kappa $\beta$; IL-18, Interleukin 18; MyD88, Myeloid differentiation primary response 88; NKGd2, Killer cell lectin-like receptor subfamily $\mathrm{K}$, member 1; HFS, high-fat sucrose diet; RSV, supplemented with trans-resveratrol $15 \mathrm{mg} / \mathrm{kg} \mathrm{BW/} \mathrm{day;} \mathrm{Q}$,

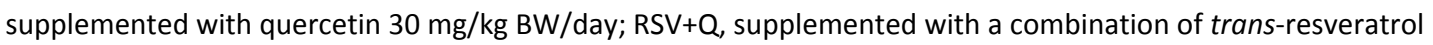
+ quercetin at the same doses.

8. Figure 8. Relative mRNA expression of tight-junction proteins and occludin in colon. Results are expressed as the mean \pm SEM. Statistical analyses were performed using Kruskal-Wallis followed by Mann Whitney $U$ test, $p$ values were corrected by Bonferroni test. ${ }^{*} p<0.05$, HFS vs RSV; ${ }^{\#}<<0.05$, HFS vs RSV+Q. TJP-1, tight-junction protein- 1; TJP-2, tight-junction protein- 2; Ocln, occludin; HFS, high-fat sucrose diet; RSV, supplemented with

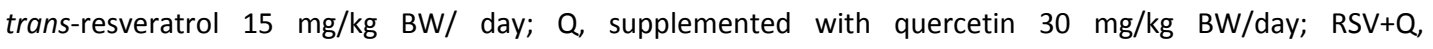
supplemented with a combination of trans-resveratrol + quercetin at the same doses. 